\title{
MONODROMY IN LOCAL GROUPS
}

\author{
C. J. ATKIN
}

(Received 6 April 1979)

Communicated by J. H. Rubinstein

\begin{abstract}
A monodromy theorem for homomorphisms of local groups into groups is proved. It follows that under suitable conditions the universal group of the local group depends only on the germ of the local group (up to natural isomorphism).
\end{abstract}

1980 Mathematics subject classification (Amer. Math. Soc.) : 22 E 05.

\section{Introduction}

The monodromy theorem of Schreier for topological groups is usually proved by means of the theory of covering spaces (see, for instance, Chevalley (1946) or Pontrjagin (1939)). Such a proof cannot apply to local groups, but it is plausible that a similar result might be proved by elementary considerations of path-deformation. In this note I construct such a proof, in some degree of generality : because of potential applications to questions of analytic extension of the Campbell-Hausdorff formula I have allowed the local groups to be asymmetric, and in view of results of Ganea (1951) I have included the possibility that the local groups are not themselves path-connected, but densely embedded in others that are. In the principal arguments I have introduced certain rather involved hypotheses of 'convexity' (see (2.8)), which seem to be useful in improving the customary numerical estimates for Banach Lie algebras, although I do not discuss that topic here.

In Section 1 I give preliminary definitions, and in Section 2 discuss technical prerequisites for the main arguments, which follow in Section 3. (Throughout the paper I have suppressed the proofs of the more mechanical and tedious auxiliary results.) In Section 4 I draw conclusions, and comment specifically on the case of local Banach Lie groups (4.6).

\section{(C) Copyright Australian Mathematical Society 1980}

Copyright. Apart from any fair dealing for scholarly purposes as permitted under the Copyright Act, no part of this JOURNAL may be reproduced by any process without written permission from the Treasurer of the Australian Mathematical Society. 


\section{Preliminaries}

(1.1) A local topological group, or local group, is a sextuple

where

$$
\Gamma=(V, e, Q, R, \mu, v)
$$

(I) $V$ is a topological space,

(II) $e \in V$ ( $e$ is the base-point of the based space $V$ ),

(III) $Q$ is an open subset of $V \times V$ and $\mu: Q \rightarrow V$ is a continuous map called 'multiplication',

(IV) $R$ is an open neighbourhood of $e$ in $V$ and $v: R \rightarrow V$ is a continuous map called 'inversion',

and such that

(V) for all $v \in V,(e, x) \in Q$ and $(x, e) \in Q$ and

$$
\mu(e, x)=\mu(x, e)=x,
$$

(VI) if $(a, b) \in Q,(b, c) \in Q,(\mu(a, b), c) \in Q,(a, \mu(b, c)) \in Q$, then

$$
\mu(a, \mu(b, c))=\mu(\mu(a, b), c),
$$

(VII) for all $x \in R,(x, v(x)) \in Q$ and $(v(x), x) \in Q$ and

$$
\mu(x, v(x))=e=\mu(v(x), x)
$$

(VIII) for all $x \in R, v(x) \in R$ and $v v(x)=x$,

(IX $\mathrm{R}_{\mathrm{R}}$ for each $x \in V$, there are open neighbourhoods $M_{e}$ of $e$ and $M_{x}$ of $x$ such that $M_{e} \times\{x\} \subseteq Q$, and the map

$$
y \mapsto \mu(y, x): M_{e} \rightarrow V
$$

maps $M_{e}$ homeomorphically onto $M_{x}$,

(IX $\mathrm{L}_{\mathrm{L}}$ for each $x \in V$, there are open neighbourhoods $N_{e}$ of $e$ and $N_{x}$ of $x$ such that $\{x\} \times N_{e} \subseteq Q$, and the map

$$
y \mapsto \mu(x, y): N_{e} \rightarrow V
$$

$$
\text { maps } N_{e} \text { homeomorphically onto } N_{x} \text {. }
$$

$\left(M_{e}\right.$ in $\left(\mathrm{IX}_{\mathrm{R}}\right)$ and $N_{e}$ in $\left(\mathrm{IX}_{\mathrm{L}}\right)$ may be called respectively right and left $x$-translation domains for $\Gamma$.)

(1.2) This definition is slightly more general than usual-compare, for example, Swierczkowski (1965) : normally one postulates symmetry, that is, that $R=V$ (the axioms (IX) are in effect substitutes for this), and also a stronger version of the associativity axiom (VI). (In fact (VI) could be weakened here to certain special cases sufficient for Lemma (2.1).) The usual axioms easily imply mine, and it is also readily 
proved that $\left(\mathrm{IX}_{\mathrm{R}}\right)$ and $\left(\mathrm{IX}_{\mathrm{L}}\right)$ are equivalent when $V$ is connected and $(\mathrm{I})-(\mathrm{VIII})$ are given.

The symbol $\Gamma$ with any affix is always to denote a local group in which the same affix is attached to every component of the sextuple. I shall regularly confuse local groups $\Gamma$ with their underlying spaces $V$, for the sake of notational convenience.

(1.3) $\Gamma_{1}$ is a local subgroup of $\Gamma$ if $V_{1}$ is a subspace of $V, e_{1}=e, Q_{1} \subseteq Q, R_{1} \subseteq R$, $\mu_{1}=\mu\left|Q_{1}, \quad v_{1}=v\right| R_{1}$. It is a full local subgroup of $\Gamma$ if in addition $Q_{1}=\left(V_{1} \times V_{1}\right) \cap \mu^{-1}\left(V_{1}\right)$. It is inversively full in $\Gamma$ if it is full and $R_{1}=R \cap V_{1} \cap v^{-1}\left(R \cap V_{1}\right)$.

A portion of $\Gamma$ is an inversively full open local subgroup. An open neighbourhood $U$ of $e$ in $\Gamma$ admits a unique local group structure making it a portion $\Gamma \mid U$ of $\Gamma$. It usually admits many weaker structures in which it is a local subgroup. Note that not every based subset of $\Gamma$ admits the structure of a local subgroup, but it must if it is open or discrete in $\Gamma$.

The local subgroup $\Gamma_{1}$ is replete in $\Gamma$ if $Q_{1}=\left(V_{1} \times V_{1}\right) \cap Q$.

(1.4) Let $\Gamma$ and $\Gamma^{\prime}$ be local groups. A homomorphism $f: \Gamma \rightarrow \Gamma^{\prime}$ is a triple $\left(f_{0}, \Gamma, \Gamma^{\prime}\right)$, where $f_{0}: V \rightarrow V^{\prime}$ is a function (not necessarily continuous) such that $f_{0}(e)=e^{\prime}$, $\left(f_{0} \times f_{0}\right) Q \subseteq Q^{\prime}, f_{0}(R) \subseteq R^{\prime}$, and $\mu^{\prime}\left(f_{0} \times f_{0}\right)=f_{0} \mu, v^{\prime} f_{0}=f_{0} v$.

In particular $\Gamma^{\prime}$ may be a group $G$, with the discrete topology and the group multiplication and inversion.

(1.5) Given a set $S$, let $S^{*}=S \times\{-1,+1\}$. Elements of $S$ are written $x^{\varepsilon} ; x \in S$ is an 'atomic term' and $\varepsilon$ is a 'formal exponent'. Define $F_{0}(S)=\emptyset, F_{n}(S)=\left(S^{*}\right)^{n}$, $F(S)=\bigcup_{n=0}^{\infty} F_{n}(S) . F(S)$, the set of unreduced associative words on $S$, admits composition by juxtaposition and the involution

$$
w \mapsto \bar{w}:\left(s_{1}^{\varepsilon_{1}}, s_{2}^{\varepsilon_{2}}, \ldots, s_{n}^{\varepsilon_{n}}\right) \mapsto\left(s_{n}^{-\varepsilon_{n}}, \ldots, s_{2}^{-\varepsilon_{2}}, s_{1}^{-\varepsilon_{1}}\right) .
$$

$c: F(S) \rightarrow F(S)$ is the operation of successively removing all 'cancelling pairs' $\left(x^{\varepsilon}, x^{-\varepsilon}\right)$ from the word $w$. The final result $c(w)$ does not depend on the choices involved, and $c(F(S))=G(S)$ is a group (the free group on $S$ ) whose multiplication is given by juxtaposition followed by cancellation. See, for instance, Kurosh (1960).

(1.6) Let now $M_{0}(S)=(\varphi, 0), M_{1}(S)=S^{*} \times\{1\}$, and thereafter inductively

$$
M_{n+1}(S)=\left[\left(M_{n}(S) \times \bigcup_{m=0}^{n} M_{m}(S)\right) \cup\left(\bigcup_{m=0}^{n-1} M_{m}(S) \times M_{n}(S)\right)\right]^{*} \times\{n+1\} .
$$

$M_{n}(S)$ is the set of nonassociative words on $S$ of 'order' $n$. The order is the last component; its purpose in the formal definition is to ensure the sets $M_{n}(S)$ are disjoint as $n$ varies, but it may evidently be ignored in notation, provided the elements of $S$ are unambiguously named by atomic terms. I shall always write, for 
instance, $\left(s_{1}^{\varepsilon_{1}}, s_{2}^{\varepsilon_{2}}\right)^{\varepsilon_{3}}$ in place of $\left(\left(\left(s_{1}^{\varepsilon_{1}}, 1\right),\left(s_{2}^{\varepsilon_{2}}, 1\right)\right)^{\varepsilon_{3}}, 2\right)$. Set now

$$
M(S)=\bigcup_{n=0}^{\infty} M_{n}(S) \text {. }
$$

There is a map $\pi: M(S) \rightarrow F(S)$ ('removal of brackets'), which is defined formally as follows : $\pi(\varphi)=\varphi, \pi\left(s^{\varepsilon}\right)=s^{\varepsilon}$; when $n$ is a positive integer and $\left(w_{1}, w_{2}\right) \in M_{n+1}(S)$,

$$
\pi\left(\left(w_{1}, w_{2}\right)^{+1}\right)=\pi\left(w_{1}\right) \cdot \pi\left(w_{2}\right), \pi\left(\left(w_{1}, w_{2}\right)^{-1}\right)=\left(\pi\left(w_{2}\right)\right)^{-} .\left(\pi\left(w_{1}\right)\right)^{-} .
$$

(The dots denote juxtaposition in $F(S)$.)

(1.7) Define $M^{r}(S) \subset M(S)$ as the set of nonassociative words of the form

$$
\left(\ldots\left(\left(\left(x_{1}^{+1}, x_{2}^{+1}\right)^{+1}, x_{3}^{+1}\right)^{+1}, \ldots, x_{n-1}^{+1}\right)^{+1}, x_{n}^{+1}\right)^{+1}
$$

(a formal inductive definition is easily supplied). These may be called right direct words. Similarly one has the set $M^{l}(S)$ of left direct words, of form $\left(x_{1}^{+1},\left(x_{2}^{+1},\left(x_{3}^{+1}, \ldots\right.\right.\right.$, $\left.\left.\left.\left(x_{n-1}^{+1}, x_{n}^{+1}\right)^{+1}\right)^{+1}\right) \ldots\right)^{+1}$. It is natural to omit the writing of formal exponents when they are +1 .

Notice that if $f: S_{1} \rightarrow S_{2}$, there are induced transformations of all the above constructions, which are inclusions when $f$ is. I shall write them in the obvious fashion : $G(f): G\left(S_{1}\right) \rightarrow G\left(S_{2}\right)$, and so on.

(1.8) Let now $\Gamma$ be a local group. Define the set ${ }^{e} M(\Gamma)$ of evaluable words in $M(\Gamma)$, and the evaluation map

$$
E(: \Gamma):{ }^{e} M(\Gamma) \rightarrow \Gamma,
$$

as follows :

(i) $\emptyset \in{ }^{e} M(\Gamma)$ and $E(\phi: \Gamma)=e$.

(ii) If $x \in V$, then $x^{\varepsilon} \in M_{1}(\Gamma)$ is evaluable in $\Gamma$ if and only if either $\varepsilon=+1$, when $E\left(x^{\varepsilon}: \Gamma\right)=x$, or $\varepsilon=-1$ and $x \in R$, when $E\left(x^{\varepsilon}: \Gamma\right)=v(x)$.

(iii) If $n>0$, then $\left(w_{1}, w_{2}\right)^{\varepsilon} \in M_{n+1}(\Gamma)$ is evaluable in $\Gamma$ if and only if both $w_{1}$ and $w_{2}$ are evaluable in $\Gamma$, and $\left(E\left(w_{1}: \Gamma\right), E\left(w_{2}: \Gamma\right)\right) \in Q$, and either $\varepsilon=+1$, in which case

$$
E\left(\left(w_{1}, w_{2}\right)^{\varepsilon}: \Gamma\right)=\mu\left(E\left(w_{1}: \Gamma\right), E\left(w_{2}: \Gamma\right)\right),
$$

or $\varepsilon=-1$ and $\mu\left(E\left(w_{1}: \Gamma\right), E\left(w_{2}: \Gamma\right)\right) \in R_{;}$in this case

$$
E\left(\left(w_{1}, w_{2}\right)^{\varepsilon}: \Gamma\right)=v\left(\mu\left(E\left(w_{1}: \Gamma\right), E\left(w_{2}: \Gamma\right)\right)\right) .
$$

Thus a word is evaluable in $\Gamma$ if it prescribes a sequence of operations that can in fact be performed in $\Gamma$. The topology of $V$ is irrelevant to this. A homomorphism $f: \Gamma_{1} \rightarrow \Gamma_{2}$ induces a transformation $M(f)$ which preserves evaluability and commutes with evaluation, that is : for any $w \in \epsilon^{e} M\left(\Gamma_{1}\right), M(f) w \in{ }^{e} M\left(\Gamma_{2}\right)$ and

$$
E\left(M(f) w: \Gamma_{2}\right)=f\left(E\left(w: \Gamma_{1}\right)\right) .
$$


As both $Q$ and $R$ are open and $\mu, v$ are continuous, it follows that any word $w \in{ }^{e} M(\Gamma)$ will remain evaluable if each atomic term suffers a sufficiently small perturbation. How small the perturbation must be to ensure this depends, of course, on $w$.

(1.9) A word $w \in F(\Gamma)$ is associative (in $\Gamma$ ) if, whenever $v_{1}, v_{2} \in M(\Gamma)$ and $\pi\left(v_{1}\right)=\pi\left(v_{2}\right)=w$, then $v_{1}, v_{2} \in^{e} M(\Gamma)$ and

$$
E\left(v_{1}: \Gamma\right)=E\left(v_{2}: \Gamma\right) \text {. }
$$

An element $w$ of $\mathrm{M}(\Gamma)$ may be called associative if $\pi(w)$ is associative. (Notice that associativity is a very strong condition; axiom VI, for instance, asserts something weaker.)

\section{Technical prerequisites}

(2.1) Lemma. Let $K_{1}, K_{2}$ be compact in $\Gamma$, with $K_{1} \times K_{2} \subseteq Q$; let $A$ be a neighbourhood of $\mu\left(K_{1} \times K_{2}\right)$ and $U$ a neighbourhood of $e$. Then there is a neighbourhood Wof e such that, for certain neighbourhoods $B_{1}$ of $K_{1}$ and $B_{2}$ of $K_{2}$, the following conditions hold.

(i) $W \subseteq R$ and $v(W)=W$.

(ii) Any word $v$ in $F_{p}(\Gamma)$ whose atomic terms all belong to $W$ with the possible exception of at most two, the exceptions having positive formal exponents and belonging to $B_{1}$ and/or $B_{2}$ in that order (that is: if there is one exception it may belong to either $B_{1}$ or $B_{2}$, but if there are two, that further to the left belongs to $B_{1}$ and that further to the right to $B_{2}$ ), is associative, for any natural number $p \leqslant 12$, and its evaluation belongs to $A$.

(iii) $E\left(\pi^{-1}\left(F_{11}(W)\right): \Gamma\right)=Z \subseteq U$.

(iv) $\mu\left(\left(Z \times K_{i}\right) \cup\left(K_{i} \times Z\right)\right) \subseteq B_{i}$ for $i=1,2$.

(v) $Z$ is both a right and a left $x$-translation domain for any $x \in \mu\left(B_{1} \times B_{2}\right)$, and

$$
\mu(W \times\{x\}) \subseteq \mu(\{x\} \times Z), \quad \mu(\{x\} \times W) \subseteq \mu(Z \times\{x\}) .
$$

The conditions given are intended to be sufficiently strong for all later applications of results of this kind. A neighbourhood of $e$ satisfying them will be called a $W\left(K_{1}, K_{2}, A, U, \Gamma\right)$.

(2.2) LemMA. Let $\Gamma$ be a local subgroup of $\tilde{\Gamma}$; suppose $K$ is compact in $\tilde{\Gamma}$, and $e \in K \subseteq \tilde{R}$. Then there is a neighbourhood $D$ of $e$ in $\tilde{\Gamma}$ such that, for certain neighbourhoods $W_{0}, W_{1}, W_{2}$ of $e$ and $A$ of $K$ in $\tilde{\Gamma}$, the following conditions are satisfied.

(i) $W_{0} \subseteq \tilde{R}$ and $\tilde{v}\left(W_{0}\right)=W_{0}$ and $A \subseteq \tilde{R}$. 
(ii) If $w_{1}, w_{2}, w_{3} \in W_{0}$ and $x \in K$, then the words in $F(\tilde{\Gamma})$

$$
\left(w_{1}, w_{2}, x, x^{-1}, w_{3}\right) \text { and }\left(w_{1}, x, x^{-1}, w_{2}, w_{3}\right)
$$

are associative.

(iii) $W_{1}$ is a $W\left(\{e\}, \tilde{v}(K), W_{0}, \tilde{\Gamma}, \tilde{\Gamma}\right)$, and $W_{2}$ is a $W\left(\{e\}, K, A, W_{1}, \tilde{\Gamma}\right)$, and $D$ is a $W\left(\{e\}, K, A, W_{2}, \tilde{\Gamma}\right)$.

(iv) $W_{1} \cap V \subseteq R$. (Note that $V$ is a subspace of $\tilde{V}$.)

(v) For any $x \in A, w \in W_{2}, \tilde{v} \tilde{\mu}(x, w) \in \tilde{\mu}\left(W_{1} \times \tilde{v}(x)\right)$ and $\tilde{v} \tilde{\mu}(w, x) \in \tilde{\mu}\left(\tilde{v}(x) \times W_{1}\right)$.

A neighbourhood $D$ of $e$ in $\tilde{\Gamma}$ satisfying the above conditions will be called a $D(K, \Gamma, \widetilde{\Gamma})$. Notice that $\Gamma$ enters only in (iv) and that a $D(K, \Gamma, \tilde{\Gamma})$ is automatically a $D(K, \tilde{\Gamma}, \tilde{\Gamma})$.

These two lemmas will be used constantly. Their proofs are easy but tedious deductions from the axioms.

(2.3) Lemma. Let $\Gamma$ be a dense replete local subgroup of $\tilde{\Gamma}$, and $x \in \Gamma$. If $U$ is a left $x$ translation domain for $\tilde{\Gamma}$, then $U \cap V$ is a left $x$-translation domain for $\Gamma$ and $\mu(\{x\} \times(U \cap V))=\tilde{\mu}(\{x\} \times U) \cap V$.

ProOF. The result is immediate if it is known that

$$
\mu(\{x\} \times(U \cap V))=\tilde{\mu}(\{x\} \times U) \cap V .
$$

By repleteness, $\{x\} \times(U \cap V) \subseteq Q$, so that certainly

$$
\mu(\{x\} \times(U \cap V)) \subseteq \tilde{\mu}(\{x\} \times U) \cap V .
$$

Suppose now that $u \in U$ and $z=\tilde{\mu}(x, u) \in V$. I show $u \in V$.

Let $T$ be a $W(\{x\},\{u\}, \tilde{\Gamma}, \tilde{\Gamma}, \tilde{\Gamma})$ such that $T \cap V$ is a $W(\{x\},\{u\}, \Gamma, \Gamma, \Gamma)$. Then $t \in T$ will lie in $V$ if and only if $\tilde{\mu}(z, t) \in V$ (since $T$ is a left $z$-translation domain for both $\Gamma$ and $\tilde{\Gamma}$, by $(2.1)(\mathrm{v}))$; or, by (2.1)(ii),

$$
t \in T \text { if and only if } \tilde{\mu}(x, \tilde{\mu}(u, t)) \in V \text {. }
$$

Suppose, if possible, that $u \notin V$. Then $\tilde{\mu}(u \times T)$ is a neighbourhood of $u$ in $\tilde{V}$ and must meet $V$. Let $y \in T$ be such that $\tilde{\mu}(u, y) \in V$. If $y \in V$, then, as $\Gamma$ is replete in $\tilde{\Gamma}$,

$$
u=\tilde{\mu}(\tilde{\mu}(u, y), v(y)) \in V .
$$

This contradicts the hypothesis, and so $y \notin V$. But $\tilde{\mu}(u, y) \in V$, and consequently, by repleteness, $\tilde{\mu}(x, \tilde{\mu}(u, y)) \in V$; however, $y \in T$, so there is a contradiction with (1). Thus $u \in V$, as required.

(2.4) Lemma. Suppose $\Gamma$ is a dense replete local subgroup of the connected local group $\tilde{\Gamma}$. Then, for any neighbourhood $U$ of $e$ in $\Gamma$,

$$
V \doteq E\left({ }^{e} M(\Gamma) \cap M^{r}(U): \Gamma\right)=E\left({ }^{e} M(\Gamma) \cap M^{l}(U): \Gamma\right)
$$


Proof. I shall prove the first equality. Suppose first that $\Gamma=\tilde{\Gamma}$, and let $\tilde{U}$ be a neighbourhood of $e$ in $\tilde{V}$. Define

$$
P=E\left({ }^{e} M(\tilde{\Gamma}) \cap M^{r}(\tilde{U}): \tilde{\Gamma}\right) .
$$

Take any $y \in \tilde{V}$, and let $\tilde{W}$ be a $W(\{e\},\{y\}, \tilde{\Gamma}, \tilde{U}, \tilde{\Gamma})$. By (2.1)(associativity and the fact that $\tilde{v}(\tilde{W})=\tilde{W})$, one sees that $z \in \tilde{\mu}(y \times \tilde{W})$ if and only if $y \in \tilde{\mu}(z \times \tilde{W})$. Thus $y \in P$ if and only if $z \in P$. But $\tilde{\mu}(y \times \tilde{W})$ is a neighbourhood of $y$; hence $P$ is open and closed in $\tilde{V}$, and, as $e \in P$, it follows that $P=\tilde{V}$.

Now suppose that $\Gamma$ is merely dense and replete in $\tilde{\Gamma}$. Let $y \in V$. Choose $\tilde{U}$ to be an open neighbourhood of $e$ in $\tilde{\Gamma}$ such that $\tilde{U} \cap V$ is included in $U$, and let $\tilde{W}$ be a $W(\{e\},\{y\}, \tilde{\Gamma}, \tilde{U}, \tilde{\Gamma})$ as before. Then, as already shown, $y$ is the evaluation in $\tilde{\Gamma}$ of a right direct word $w$ on $\tilde{U}$. Perturb each atomic term of $w$ so as to lie in $U$, but by so little that the perturbed word $w^{\prime}$ remains in $M^{r}(\tilde{U}) \cap{ }^{e} M(\tilde{\Gamma})$ and

$$
x=E\left(w^{\prime}: \tilde{\Gamma}\right) \in \tilde{\mu}(y \times \tilde{W}) .
$$

Thus there is $q \in \tilde{W}$ such that $y=\tilde{\mu}(x, q)$, by (2.1)(i),(ii). Since $\Gamma$ is replete in $\tilde{\Gamma}$ and $w^{\prime}$ is a word on $V$ evaluable in $\tilde{\Gamma}$, it is evaluable in $\Gamma$ and $x \in V$. But now by (2.3), $q \in \tilde{W} \cap V$, and so

$$
y=E\left(\left(w^{\prime}, q\right): \Gamma\right),
$$

again by repleteness. As $\tilde{W} \cap V \subseteq U$, this completes the proof.

(2.5) Lemma. Suppose $e \in K \subseteq R$ and $K$ is compact. Let Wbe a $D(K, \Gamma, \Gamma)$. Then, for each $x \in \mu((K \times W) \cup(W \times K))$ and $w \in W$,

$$
v(\mu(w, x))=\mu(v(x), v(w)) \quad \text { and } \quad v(\mu(x, w))=\mu(v(w), v(x)) .
$$

Proof. By construction, both sides of the equation make sense. From property (2.2)(v) (with $W_{1}$ as there introduced) $\nu \mu(x) \in \mu\left(v(x) \times W_{1}\right)$, so that $v \mu(w, x)=\mu\left(v(x), w_{1}\right)$ for some unique $w_{1}$ in $W_{1}$. Hence

$$
\begin{aligned}
e & =\mu\left(\mu(w, x), \mu\left(v(x), w_{1}\right)\right) \\
& =\mu\left(w, \mu\left(x, \mu\left(v(x), w_{1}\right)\right)\right) \\
& =\mu\left(w, \mu\left(\mu(x, v(x)), w_{1}\right)\right)=\mu\left(w, w_{1}\right),
\end{aligned}
$$

so that

as required.

$$
\begin{aligned}
v(w) & =\mu\left(v(w), \mu\left(w, w_{1}\right)\right) \\
& =\mu\left(\mu(v(w), w), w_{1}\right)=w_{1},
\end{aligned}
$$

(2.6) Lemma. Suppose $K$ is a connected compact subset of $\tilde{R}$ containing e, and $\Gamma$ is a dense replete local subgroup of $\tilde{\Gamma}$. Let $W$ be a $D(K, \Gamma, \tilde{\Gamma})$. Then $\tilde{v}(V \cap \tilde{\mu}(W \times K)) \subseteq V$. 
Proof. Consider $P=\{x \in K: \tilde{v}(V \cap \tilde{\mu}(W \times x)) \quad V\}$. If, for $i=1,2$, $a_{i}=\tilde{\mu}\left(w_{i}, x\right) \in V \cap \tilde{\mu}(W \times x)$ where $w_{i} \in W$, then $a_{2}=\tilde{\mu}\left(w, a_{1}\right)$, where $w=\tilde{\mu}\left(w_{2}, \tilde{v}\left(w_{1}\right)\right)$. Taking $U=\tilde{\mu}(W \times W)$ in (2.3), it follows that $w \in V$. Now by (2.5) $\tilde{v}\left(a_{2}\right)=\tilde{\mu}\left(\tilde{v}\left(a_{1}\right), \tilde{v}(w)\right)$, where, as $w \in R, \tilde{v}(w) \in V$. By repleteness, $\tilde{v}\left(a_{2}\right) \in V$ whenever $\tilde{v}\left(a_{1}\right) \in V$. Hence, in fact, $\tilde{v}(V \cap \tilde{\mu}(W \times x)) \cap V \neq \emptyset$ only if $\tilde{v}(V \cap \tilde{\mu}(W \times x)) \subseteq V$. Thus $P$ is open and closed in $K$. Since, evidently, $e \in P$, it follows that $P=K$ as required.

(The argument also shows that $\tilde{v}\left(\tilde{R}_{0} \cap V\right) \subseteq V$, if $\widetilde{R}_{0}$ is the component of $e$ in $\tilde{R}$.)

(2.7) Establish once and for all the following notation : reading $\subseteq$ to mean is a local subgroup of', I always assume

$$
\begin{aligned}
& \tilde{\Gamma} \subseteq \tilde{\Gamma}^{0} \subseteq \tilde{\Gamma}^{1} \subseteq \tilde{\Gamma}^{2} \\
& \text { UI Ul UI UI } \\
& \Gamma \subseteq \Gamma^{0} \subseteq \Gamma^{1} \subseteq \Gamma^{2}
\end{aligned}
$$

where $\Gamma$ is dense in $\tilde{\Gamma}, \Gamma^{0}$ in $\tilde{\Gamma}^{0}$, and likewise $\Gamma^{1}$ in $\tilde{\Gamma}^{1}, \Gamma^{2}$ in $\tilde{\Gamma}^{2}$.

In much of what follows, definitions and theorems have both right- and lefthanded versions. In general, I shall state and prove only one version.

(2.8) Definitions. (i) $\Gamma^{0}$ is (left) 0 -convex in $\left(\tilde{\Gamma}^{0}, \tilde{\Gamma}^{1}, \tilde{\Gamma}^{2}\right)$ if, whenever $q: I \rightarrow \tilde{V}^{0}$ is a path with $q(0)=e, q(1)=y \in V^{0}$, and $(x, y) \in Q^{0}$, there is a path $p: I \rightarrow \tilde{V}^{0}$ such that $p(0)=e, p(1)=y, x \times p(I) \subseteq \widetilde{Q}^{1}$, and $p$ and $q$ are homotopic as paths from $e$ to $y$ in $\tilde{V}^{2}$.

(ii) $\Gamma^{0}$ is (left) 1 -convex in $\left(\tilde{\Gamma}^{0}, \tilde{\Gamma}^{1}, \tilde{\Gamma}^{2}\right)$ if, whenever $p$ and $q$ are paths in $\tilde{V}^{0}$ such that $p(0)=q(0)=e$ and

$$
(p(1), q(1)) \in Q^{0}, \quad p(1) \times q(I) \subseteq \widetilde{Q}^{1},
$$

and $r: I \rightarrow \tilde{V}^{1}$ is defined by $: r(t)=p(2 t)$ for $0 \leqslant t \leqslant \frac{1}{2}, r(t)=\tilde{\mu}^{1}(p(1), q(2 t-1))$ for $\frac{1}{2} \leqslant t \leqslant 1$, - then there is a path $s: I \rightarrow \widetilde{V}^{0}$ with $s(0)=e$ and $s(1)=x=r(1)$, such that $s$ and $r$ are homotopic as paths from $e$ to $x$ in $\tilde{V}^{2}$.

(iii) $\Gamma^{0}$ is inversively convex in $\left(\tilde{\Gamma}^{0}, \tilde{\Gamma}^{2}\right)$ if, whenever $p$ is a path $I \rightarrow \tilde{V}^{0}$ such that $p(0)=e$ and $p(1)=x \in R^{0}$, there exist paths $q: I \rightarrow \widetilde{R}^{2}$ and $r: I \rightarrow \tilde{V}^{0}$ such that, firstly, $q(0)=e, q(1)=x$, and $q, p$ are homotopic as paths from $e$ to $x$ in $\tilde{V}^{2}$; and, secondly, $r(0)=e, r(1)=v^{0}(x)$, and $r, \tilde{v}^{2} \circ q$ are homotopic as paths between $e$ and $v^{0}(x)$ in $V^{2}$.

(iv) $\Gamma^{0}$ is (left) convex in $\left(\Gamma^{0}, \Gamma^{1}, \tilde{\Gamma}^{2}\right)$ if it is (left) 0 -convex and 1-convex in $\left(\tilde{\Gamma}^{0}, \tilde{\Gamma}^{1}, \tilde{\Gamma}^{2}\right)$ and inversively convex in $\left(\tilde{\Gamma}^{0}, \tilde{\Gamma}^{2}\right)$.

These conditions set out precisely what is used in the sequel; but, despite their complexity, they should not be substituted too lightly by the simplified conditions I give next. There are circumstances, in local Lie groups for instance, where the simplifications can lead to much weaker results. 
(2.9) The based subspace $(X, e)$ of the based space $(Y, e)$ may be called pathconnected in $Y$ if every point in $X$ may be connected to $e$ by a path in $Y$. Likewise $X$ is simply-connected in $Y$ if every loop at $e$ in $X$ contracts to a point in $Y$.

(2.10) Lemma. (i) $\Gamma^{0}$ is (left) 0-convex in $\left(\tilde{\Gamma}^{0}, \tilde{\Gamma}^{1}, \tilde{\Gamma}^{2}\right)$ whenever $\tilde{V}^{0} \times \tilde{V}^{0} \subseteq \tilde{Q}^{1}$.

(ii) $\Gamma^{0}$ is (left) 1-convex in $\left(\tilde{\Gamma}^{0}, \tilde{\Gamma}^{1}, \tilde{\Gamma}^{2}\right)$ whenever $V^{0}$ is path-connected in $\tilde{V}^{0}$ and $\tilde{V}^{1}$ is simply-connected in $\tilde{V}^{2}$.

(iii) $\Gamma^{0}$ is inversively convex in $\left(\tilde{\Gamma}^{0}, \tilde{\Gamma}^{2}\right)$ if $R^{0}$ is path-connected in $\tilde{R}^{1}$ and $\tilde{V}^{1}$ is simply-connected in $\tilde{V}^{2}$.

(iv) $\Gamma^{0}$ is (left) convex in $\left(\tilde{\Gamma}^{0}, \tilde{\Gamma}^{1}, \tilde{\Gamma}^{2}\right)$ if $V^{0}$ is path-connected in $\tilde{V}^{0}, R^{0}$ is pathconnected in $\tilde{R}^{1}, \tilde{V}^{0} \times \tilde{V}^{0} \subseteq \tilde{Q}^{1}$, and $\tilde{V}^{1}$ is simply-connected in $\tilde{V}^{2}$.

(2.11) Definition. Suppose $p: I \rightarrow \tilde{V}$ is a path with $p(0)=x \in V$ and $p(1)=y \in V$, and let $\tilde{U}$ be a neighbourhood of $e$ in $\tilde{V}$. Then a $\tilde{U}$-approximation of $p$ in $V$ is a finite sequence $\left(\left(t_{i}, \alpha_{i}\right)\right)_{0 \leqslant i \leqslant m}$, for some natural number $m$, where, for each $i, t_{i} \in I$ and $\alpha_{i} \in V$, and $0=t_{0}<t_{1}<t_{2}<\ldots<t_{m}=1, \alpha_{0}=x, \alpha_{m}=y$, and for $1 \leqslant i<m$,

$$
\alpha_{i} \in V \cap \bigcap_{t \in\left[t_{i-1}, t_{i+1}\right]} \tilde{\mu}((p(t) \times \tilde{U}) \cap \tilde{Q}),
$$

whilst

$$
x=\alpha_{0} \in V \cap \bigcap_{t \in\left[0, t_{1}\right]} \tilde{\mu}((p(t) \times \tilde{U}) \cap \tilde{Q})
$$

and

$$
y=\alpha_{m} \in V \cap \bigcap_{t \in\left[t_{m-1}, 1\right]} \tilde{\mu}((p(t) \times \tilde{U}) \cap \tilde{Q}) .
$$

(Strictly speaking, this is a left $\tilde{U}$-approximation of $p$ in $V$. Note that a $\tilde{U}$ approximation of $p$ in $V$ is automatically a $\tilde{U}_{1}$-approximation if $\tilde{U} \subseteq \tilde{U}_{1}$.)

(2.12) Given $p$ and $\tilde{U}$ as above, there always exists a $\tilde{U}$-approximation of $p$ in $V$. Let $\tilde{U}_{0}$ be a $W(p(I),\{e\}, \tilde{V}, \tilde{U}, \tilde{\Gamma})$; then the class

$$
\left\{\operatorname{int}_{\tilde{V}} \tilde{\mu}\left(p(s) \times \tilde{U}_{0}\right): s \in[0,1]\right\}
$$

is an open cover of $p(I)$. Choose $0=t_{0}<t_{1}<\ldots<t_{m}=1$ such that, for each $i$, $0 \leqslant i<m$, there is some $s_{i} \in[0,1]$ with

$$
p\left[t_{i}, t_{i+1}\right] \subseteq \operatorname{int}_{\bar{V}} \tilde{\mu}\left(p\left(s_{i}\right) \times \tilde{U}_{0}\right) .
$$

Now let $\alpha_{0}=x, \alpha_{m}=y$, and, for $1 \leqslant i<m$, choose

This suffices.

$$
\alpha_{i} \in V \cap \tilde{\mu}\left(p\left(s_{i-1}\right) \times \tilde{U}_{0}\right) \cap \tilde{\mu}\left(p\left(s_{i}\right) \times \tilde{U}_{0}\right) .
$$

For brevity I shall say that $\left(\left(t_{i}, \alpha_{i}\right)\right)_{0 \leqslant i \leqslant m}$ is a fine $\tilde{U}$-approximation of p in Vif it is in fact a $\tilde{U}_{1}$-approximation for some set $\tilde{U}_{1}$ which is a $W(p(I),\{e\}, \tilde{V}, \tilde{U}, \tilde{\Gamma})$. Then $(2.1)(\mathrm{v})$ and (2.3) lead to the conclusion that, if $\Gamma$ is replete in $\tilde{\Gamma}$ and $\left(\left(t_{i}, \alpha_{i}\right)\right)_{0 \leqslant i \leqslant m}$ is a fine $\tilde{U}$ approximation of $p$ in $V$, there are, for each relevant $i$, unique points $\beta_{i}$ and $\beta_{i}^{\prime}$ in 
$\tilde{U} \cap V\left(\right.$ in fact in $\left.\tilde{\mu}\left(\tilde{U}_{1} \times \tilde{U}_{1}\right) \cap V\right)$ such that

$$
\alpha_{i+1}=\mu\left(\alpha_{i}, \beta_{i}\right)=\mu\left(\beta_{i}^{\prime}, \alpha_{i}\right) .
$$

The words

$$
\left(\left(\left(\ldots\left(\beta_{0}, \beta_{1}\right), \beta_{2}\right), \ldots\right), \beta_{m-1}\right) \text { and }\left(\beta_{m-1}^{\prime},\left(\beta_{m-2}^{\prime},\left(\ldots\left(\beta_{1}^{\prime}, \beta_{0}^{\prime}\right)\right) \ldots\right)\right)
$$

are respectively the right and left direct words (in $M(\tilde{U} \cap V))$ associated with the $\tilde{U}$ approximation.

(2.13) Proposition. Suppose $p: I \rightarrow \tilde{V}^{0}$ is a path with end-points $e$, a in $V^{0}$. Let $\Gamma$ be full in $\tilde{\Gamma}$, which is open in $\tilde{\Gamma}^{0}$; let $\Gamma^{0}$ be replete in $\tilde{\Gamma}^{0}$, and let $V=\tilde{V} \cap V^{0}$. Suppose $g: \Gamma \rightarrow G$ is a homomorphism of $\Gamma$ into a group $G$.

Let $\tilde{U}$ be a neighbourhood of $e$ in $\tilde{V}$ such that $\tilde{U} \times \tilde{U} \subseteq \tilde{Q}$, and suppose $w_{1}, w_{2}$ are right direct words associated with fine $\tilde{U}$-approximations of $p$ in $V^{0}$ and $w_{3}$ is the left direct word associated with some further fine $\tilde{U}$-approximation of $p$ in $V^{0}$. Then $w_{1}, w_{2}$ and $w_{3}$ lie in $M(\Gamma)$ and

$$
E\left(M(g) w_{1}: G\right)=E\left(M(g) w_{2}: G\right)=E\left(M(g) w_{3}: G\right) .
$$

Proof. As $V=\tilde{V} \cap V^{0}$, the assertion that $w_{1}, w_{2}, w_{3} \in M(\Gamma)$ follows from the construction (2.12). I begin by proving the first equality.

(2.14) Suppose that $w_{j}$ (where $j=1,2$ ) is associated with the $\tilde{W}_{j}$-approximation $\left(\left(t_{i}^{j}, \alpha_{i}^{j}\right)\right)_{0 \leqslant i \leqslant m j}$ of $p$ in $V^{0}$, where $\tilde{W}_{j}$ is a $W\left(p(I),\{e\}, \widetilde{V}^{0}, \tilde{U}, \Gamma^{0}\right)$. I may clearly construct, as in (2.12), a $\tilde{W}_{1} \cap \tilde{W}_{2}$-approximation $\left(\left(t_{i}, \alpha_{i}\right)\right)_{0 \leqslant i \leqslant m}$ such that the subdivision points $\left(t_{i}\right)$ include all the $\left(t_{i}^{j}\right)$. Thus I may assume in the proof, without any loss of generality, that $\tilde{W}_{1}=\tilde{W}_{2}=\tilde{W}_{0}$ and that every $t_{i}^{1}$ is a $t_{k}^{2}$; in fact I may write $t_{i}^{1}=t_{k(i)}^{2}$, where $k(0)=0, k(i)<k(i+1)$ for each $i$, and $k\left(m_{1}\right)=m_{2}$.

Now, given $i, 0 \leqslant i<m_{1}$, and $k(i) \leqslant k<k(i+1)$, let $\xi_{k}$ be the unique point of $\tilde{U}$ such that $\alpha_{k}^{2}=\tilde{\mu}^{0}\left(\alpha_{i}^{1}, \xi_{k}\right)$. (Thus $\xi_{0}=\xi_{m_{2}}=e$.) When $k=k(i+1)$, let $\xi_{k(i+1)}^{\prime}$ be the unique element of $U$ with

$$
\alpha_{k(i+1)}^{2}=\tilde{\mu}^{0}\left(\alpha_{i}^{1}, \xi_{k(i+1)}^{\prime}\right) .
$$

By construction (compare (2.1), (2.12), (2.3)), the points $\xi_{k}, \xi_{k(i+1)}^{\prime}$ all lie in $\tilde{U} \cap V^{0}$, and so in $V$.

But, when $k(i) \leqslant k<k(i+1)$,

$$
\begin{aligned}
\tilde{\mu}^{0}\left(\alpha_{i}^{1}, \xi_{k+1}\right) & =\alpha_{k+1}^{2}=\tilde{\mu}^{0}\left(\alpha_{k}^{2}, \beta_{k}^{2}\right) \\
& =\tilde{\mu}^{0}\left(\tilde{\mu}^{0}\left(\alpha_{i}^{1}, \xi_{k}\right), \beta_{k}^{2}\right) \\
& =\tilde{\mu}^{0}\left(\alpha_{i}^{1}, \tilde{\mu}^{0}\left(\xi_{k}, \beta_{k}^{2}\right)\right)
\end{aligned}
$$


by virtue of the associativity guaranteed in (2.1). Hence

$$
\xi_{k+1}=\tilde{\mu}^{0}\left(\xi_{k}, \beta_{k}^{2}\right)=\tilde{\mu}\left(\xi_{k}, \beta_{k}^{2}\right)=\mu\left(\xi_{k}, \beta_{k}^{2}\right),
$$

since, in the first place, $\xi_{k}$ and $\beta_{k}^{2}$ lie in $U$, so that their product is defined in $\tilde{\Gamma}$, and, secondly, $\xi_{k+1}, \xi_{k}, \beta_{k}^{2}$ all belong to $V$, which means I can apply fullness of $\Gamma$ in $\tilde{\Gamma}$ to write $\mu$ instead of $\tilde{\mu}$.

When $k=k(i+1)-1$, likewise

$$
\xi_{k(i+1)}^{\prime}=\mu\left(\xi_{k(i+1)-1}, \beta_{k(i+1)-1}^{2}\right)=\mu\left(\beta_{i}^{1}, \xi_{k(i+1)}\right) .
$$

Now define, for $0<i \leqslant m_{1}$,

$$
\begin{aligned}
& w_{1}^{(i)}=\left(\left(\ldots\left(\beta_{0}^{1}, \beta_{1}^{1}\right), \ldots\right), \beta_{i-1}^{1}\right), \\
& w_{2}^{(i)}=\left(\left(\ldots\left(\beta_{0}^{2}, \beta_{1}^{2}\right), \ldots\right), \beta_{k(i)-1}^{2}\right),
\end{aligned}
$$

and, for $i=0$, let $w_{1}^{(0)}=w_{2}^{(0)}$ be the empty word.

Suppose $0 \leqslant r<m_{1}$ and $E\left(M(g)\left(w_{1}^{(r)}, \xi_{k(r)}\right): G\right)=E\left(M(g) w_{2}^{(r)}: G\right)$. Then

$$
\begin{aligned}
E\left(M(g) w_{2}^{(r+1)}: G\right) & =E\left(\left(M(g)\left(\left(\ldots\left(w_{2}^{(r)}, \beta_{k(r)}^{2}\right), \ldots\right), \beta_{k(r+1)-1}^{2}\right)\right): G\right) \\
& =E\left(M(g) w_{2}^{(r)}: G\right) \cdot E\left(\left(M(g)\left(\left(\ldots\left(\beta_{k(r)}^{2}, \beta_{k(r)+1}^{2}\right), \ldots\right), \beta_{k(r+1)-1}^{2}\right)\right): G\right) \\
& =E\left(M(g)\left(w_{1}^{(r)}, \xi_{k(r)}\right): G\right) \cdot E\left(M(g)\left(\left(\ldots\left(\beta_{k(r)}^{2}, \ldots\right), \beta_{k(r+1)-1}^{2}\right)\right): G\right) \\
& =E\left(M(g) w_{1}^{(r)}: G\right) . E\left(M(g)\left(\left(\ldots\left(\xi_{k(r)}, \beta_{k(r)}^{2}\right), \ldots\right), \beta_{k(r+1)-1}^{2}\right): G\right) .
\end{aligned}
$$

However, $g: \Gamma \rightarrow G$ is a homomorphism; consequently by (1) above

$$
g\left(\xi_{k(r)}\right) \cdot g\left(\beta_{k(r)}^{2}\right)=g \mu\left(\xi_{k(r)}, \beta_{k(r)}^{2}\right)=g\left(\xi_{k(r)+1}\right)
$$

and so on inductively. Eventually

$$
\begin{aligned}
E\left(M(g) w_{2}^{(r+1)}: G\right) & =E\left(M(g) w_{1}^{(r)}: G\right) \cdot g\left(\xi_{k(r+1)-1}\right) \cdot g\left(\beta_{k(r+1)-1}^{2}\right) \\
& =E\left(M(g) w_{1}^{(r)}: G\right) \cdot g\left(\beta_{r}^{1}, \xi_{k(r+1)}\right) \text { by }(2) \\
& =E\left(M(g)\left(w_{1}^{(r)}, \beta_{r}^{1}\right): G\right) \cdot g\left(\xi_{k(r+1)}\right) \\
& =E\left(M(g) w_{1}^{(r+1)}: G\right) \cdot g\left(\xi_{k(r+1)}\right) \\
& =E\left(M(g)\left(w_{1}^{(r+1)}, \xi_{k(r+1)}\right): G\right) .
\end{aligned}
$$

Hence by induction (the case $r=0$ being evident)

$$
E\left(M(g)\left(w_{1}^{(r)}, \xi_{k(r)}\right): G\right)=E\left(M(g) w_{2}^{(r)}: G\right)
$$

for all $r, 0 \leqslant r \leqslant m_{1}$. When $r=m_{1}$ this shows

$$
E\left(M(g) w_{1}: G\right)=E\left(M(g) w_{2}: G\right)
$$


(2.15) Now I prove the second equality of (2.13). From what has already been shown in (2.14) (and the analogous result for associated right direct words, whose demonstration is here omitted) it is apparent that it suffices to prove the existence of one fine $\tilde{U}$-approximation of $p$ in $V^{0}$ such that, if $w_{1}, w_{3}$ are the associated right and left direct words respectively,

$$
E\left(M(g) w_{1}: G\right)=E\left(M(g) w_{3}: G\right) .
$$

Given a fine $\tilde{U}$-approximation $\left(\left(t_{i}, \alpha_{i}^{\prime}\right)_{0 \leqslant i \leqslant m}\right.$ of $p$ in $V^{0}$, that is, a $\tilde{U}_{0^{-}}$ approximation, where $\widetilde{U}_{0}$ is a $W\left(p(I),\{e\}, \widetilde{V}^{0}, \tilde{U}, \widetilde{\Gamma}^{0}\right)$, there is for each $i, 0 \leqslant i<m$, a unique continuous path

$$
p_{i}: I \rightarrow \tilde{\mu}^{0}\left(\tilde{U}_{0} \times \tilde{U}_{0}\right)
$$

such that for all $\tau \in \mathbf{I}$

$$
p\left(\tau t_{i+1}+(1-\tau) t_{i}\right)=\tilde{\mu}^{0}\left(p\left(t_{i}\right), p_{i}(\tau)\right) .
$$

Let $\tilde{U}_{i+1}$ be defined inductively as a $W\left(p_{i}(I),\{e\}, \tilde{V}^{0}, \tilde{U}_{i}, \tilde{\Gamma}^{0}\right)$; choose a $\tilde{U}_{m^{-}}$ approximation $\left(\left(\tau_{j}, \alpha_{j}\right)\right)_{0 \leqslant j \leqslant n}$ of $p$ in $V^{0}$ so that, for $0 \leqslant i \leqslant m, t_{i}=\tau_{j(i)}$ (where $j(0)=0$, $j(m)=n$, and $j$ is strictly increasing) and $\left(\left(\tau_{j(i)}, \alpha_{j(i)}\right)\right)_{0 \leqslant i \leqslant m}$ is simultaneously a fine $\tilde{U}$ approximation of $p$ in $V^{0}$. This is clearly possible, as $V^{0}$ is dense in $\tilde{V}^{0}$.

Let

$$
\left(\left(\left(\ldots\left(\beta_{0}, \beta_{1}\right), \beta_{2}\right), \ldots\right), \beta_{m-1}\right)
$$

be the right direct word associated with $\left(\left(t_{i}, \alpha_{j(i)}\right)\right)$,

$$
\left(\left(\left(\ldots\left(\gamma_{0}, \gamma_{1}\right), \gamma_{2}\right), \ldots\right), \gamma_{n-1}\right)
$$

be the right direct word associated with $\left(\left(\tau_{j}, \alpha_{j}\right)\right)$,

$$
\left(\delta_{n-1},\left(\ldots,\left(\delta_{1}, \delta_{0}\right)\right) \ldots\right)
$$

be the left direct word associated with $\left(\left(\tau_{j}, \alpha_{j}\right)\right)$. Write also, for $0 \leqslant k<n$,

$$
\begin{array}{ll}
w_{1}^{(k)}=\left(\left(\ldots\left(\gamma_{0}, \gamma_{1}\right), \ldots\right), \gamma_{k}\right) & \left(\text { notice } w_{1}^{(n-1)}=w_{1}\right), \\
\left.w_{3}^{(k)}=\left(\delta_{k}, \ldots,\left(\delta_{1}, \delta_{0}\right)\right) \ldots\right) & \left(\text { notice } w_{3}^{(n-1)}=w_{3}\right) .
\end{array}
$$

Thus $E\left(M(g) w_{1}^{(0)}: G\right)=E\left(M(g) w_{3}^{(0)}: G\right)$, since $\delta_{0}=\gamma_{0}=\alpha_{0}$.

Suppose now that $0 \leqslant q<m$ and $j(q) \leqslant k<j(q+1)$, and define uniquely $\alpha_{l}^{q} \in \tilde{\mu}^{0}\left(\tilde{U}_{0} \times \tilde{U}_{0}\right)$ and $\tau_{l}^{q} \in I$, for $0 \leqslant l \leqslant j(q+1)-j(q)$, by

$$
\begin{aligned}
\alpha_{j(q)+l} & =\tilde{\mu}^{0}\left(\alpha_{j(q)}, \alpha_{l}^{q}\right), \\
\tau_{j(q)+l} & =t_{q}+\tau_{l}^{q}\left(t_{q+1}-t_{q}\right) .
\end{aligned}
$$

Then the homeomorphisms assured by the construction (cf. (2.1)) show that $\left(\left(\tau_{l}^{q}, \alpha_{l}^{q}\right)\right)$ is a fine $\tilde{U}$-approximation of $p_{q}$, and that the associated right direct word is 
$\left(\left(\ldots\left(\gamma_{j(q)}, \gamma_{j(q)+1}\right), \ldots\right), \gamma_{j(q+1)-1}\right)$. This word is therefore evaluable in $\tilde{\Gamma}$ (cf. (2.12)), and, as all the terms and all the partial products lie in $V$, it is in fact evaluable in $\Gamma$.

Let $\rho_{l}^{q}=E\left(\left(\left(\ldots\left(\gamma_{j(q)}, \gamma_{j(q)+1}\right), \ldots\right), \gamma_{j(q)+l}\right): \Gamma\right)$. If $l=0$, then $\alpha_{j(q)+1}=\tilde{\mu}^{0}\left(\alpha_{j(q)}, \rho_{0}^{q}\right)$ by the definitions. Suppose $l<j(q+1)-j(q)-1$ and $\alpha_{j(q)+l+1}=\tilde{\mu}^{0}\left(\alpha_{j(q)}, \rho_{l}^{q}\right)$. Then

$$
\begin{aligned}
\alpha_{j(q)+l+2} & =\tilde{\mu}^{0}\left(\alpha_{j(q)+l+1}, \gamma_{j(q)+l+1}\right) \\
& =\tilde{\mu}^{0}\left(\tilde{\mu}^{0}\left(\alpha_{j(q)}, \rho_{l}^{q}\right), \gamma_{j(q)+l+1}\right) \\
& =\tilde{\mu}^{0}\left(\alpha_{j(q)}, \tilde{\mu}^{0}\left(\rho_{l}^{q}, \gamma_{j(q)+l+1}\right)\right) \\
& =\tilde{\mu}^{0}\left(\alpha_{j(q)}, \rho_{l+1}^{q}\right),
\end{aligned}
$$

by virtue of the associativities given by the construction. The products actually exist in $\Gamma$, so we may write $\mu$ in place of $\tilde{\mu}^{0}$.

Next, by construction of $\tilde{U}_{m}$, there must be a unique element of $\tilde{U}_{q}$, say $\gamma_{j(q)+1+1}^{0}$, such that

$$
\tilde{\mu}^{0}\left(\gamma_{j(q)+l+1}^{0}, \rho_{l}^{q}\right)=\tilde{\mu}^{0}\left(\rho_{l}^{q}, \gamma_{j(q)+l+1}\right)=\mu\left(\rho_{l}^{q}, \gamma_{j(q)+l+1}\right)
$$

(as above. For the existence, cf. (2.1)(v).) As $\Gamma^{0}$ is replete in $\tilde{\Gamma}^{0}, \gamma_{j(q)+l+1}^{0} \in V^{0}(\operatorname{see}(2.3)$ ) and so $\gamma_{j(q)+l+1}^{0} \in \tilde{U}_{q} \cap V$, when we recall that $V^{0} \cap \tilde{V}=V$.

In turn there is a unique $\gamma_{j(q)+1+1}^{1} \in \tilde{U}_{q-1}$ such that

$$
\tilde{\mu}^{0}\left(\gamma_{j(q)+l+1}^{1}, \beta_{q-1}\right)=\tilde{\mu}^{0}\left(\beta_{q-1}, \gamma_{j(q)+l+1}^{0}\right)=\mu\left(\beta_{q-1}, \gamma_{j(q)+l+1}^{0}\right) .
$$

(Certainly $\beta_{q-1} \times \tilde{U}_{q} \subseteq \check{Q}$ by construction; as $\Gamma^{0}$ is replete in $\tilde{\Gamma}^{0}$,

$$
\tilde{\mu}^{0}\left(\beta_{q-1}, \gamma_{j(q)+l+1}^{0}\right) \in V^{0} \cap \tilde{V}=V ;
$$

fullness of $\Gamma$ in $\tilde{\Gamma}$ shows the product may be taken in $\Gamma)$. By $(2.3), \gamma_{j(q)+l+1}^{1} \in \tilde{U}_{q-1} \cap V$. This procedure may evidently be repeated, giving $\gamma_{j(q)+l+1}^{t} \in \tilde{U}_{q-t} \cap V$ for $0<t \leqslant q$, such that $\mu\left(\gamma_{j(q)+l+1}^{t}, \beta_{q-t}\right)=\mu\left(\beta_{q-t}, \gamma_{j(q)+l+1}^{t-1}\right)$.

Now suppose that $E\left(M(g) w_{1}^{(k)}: G\right)=E\left(M(g) w_{3}^{(k)}: G\right)$. Then

$$
\begin{aligned}
E\left(M(g) w_{1}^{(k+1)}: G\right)= & \left.\left.E\left(M(g)\left(\left(\ldots\left(w_{1}^{(j(q)-1)}, \gamma_{j(q)}\right), \ldots\right), \gamma_{k}\right), \gamma_{k+1}\right)\right): G\right) \\
= & \left.E\left(M(g) w_{1}^{(j(q)-1}: G\right) \cdot g\left(E\left(\left(\ldots\left(\gamma_{j(q)}, \gamma_{j(q)+1}\right), \ldots\right), \gamma_{k}\right)\right): \Gamma\right) \cdot g\left(\gamma_{k+1}\right) \\
& \quad(\text { since } g \text { is a homomorphism; recall (1) above) } \\
= & E\left(M(g) w_{1}^{(j(q)-1)}: G\right) \cdot g\left(\rho_{k-j(q)}^{q}\right) \cdot g\left(\gamma_{k+1}\right) \\
= & E\left(M(g) w_{1}^{(j(q)-1)}: G\right) \cdot g\left(\mu\left(\rho_{k-j(q)}^{q}, \gamma_{k+1}\right)\right) \\
= & E\left(M(g) w_{1}^{(j(q)-1)} ; G\right) \cdot g\left(\mu\left(\gamma_{k+1}^{0}, \rho_{k-j(q)}^{q}\right)\right) \\
= & E\left(\left(M(g)\left(\left(\ldots\left(w_{1}^{j(q-1)-1)}, \gamma_{j(q-1)}\right), \ldots\right), \gamma_{j(q)-1}\right)\right): G\right) \\
& \quad \cdot g\left(\gamma_{k+1}^{0}\right) \cdot g\left(\rho_{k-j(q)}^{q}\right)
\end{aligned}
$$




$$
\begin{gathered}
=E\left(M(g) w_{1}^{(j(q-1)-1)}: G\right) \cdot g\left(\beta_{q-1}\right) \cdot g\left(\gamma_{k+1}^{0}\right) \\
. E\left(M(g)\left(\left(\ldots\left(\gamma_{j(q)}, \ldots\right), \gamma_{k}\right)\right): G\right)
\end{gathered}
$$

(by the same argument as before)

$$
=E\left(M(g) w_{1}^{(j(q-1)-1)}: G\right) \cdot g\left(\mu\left(\gamma_{k+1}^{1}, \beta_{q-1}\right)\right) \cdot E\left(M(g)\left(\left(\ldots\left(\gamma_{j(q)}, \ldots\right), \gamma_{k}\right)\right): G\right)
$$

and so on, passing $\gamma_{k+1}$ across the $\beta$ 's one by one, until finally

$$
\begin{aligned}
= & \left.g\left(\gamma_{k+1}^{q}\right) \cdot E\left(M(g)\left(\left(\ldots\left(\gamma_{0}, \gamma_{1}\right), \ldots\right), \gamma_{j(q)-1}\right)\right): G\right) \\
& \quad \cdot E\left(M(g)\left(\left(\ldots\left(\gamma_{j(q)}, \gamma_{j(q)+1}\right), \ldots\right), \gamma_{k}\right): G\right) \\
= & g\left(\gamma_{k+1}^{q}\right) \cdot E\left(M(g) w_{1}^{(k)}: G\right) \\
= & g\left(\gamma_{k+1}^{q}\right) \cdot E\left(M(g) w_{3}^{(k)}: G\right) \\
= & E\left(M(g)\left(\gamma_{k+1}^{q}, w_{3}^{(k)}\right): G\right) . \quad \text { (by the inductive hypothesis) }
\end{aligned}
$$

Thus the inductive step, showing that

$$
E\left(M(g) w_{1}^{(k+1)}: G\right)=E\left(M(g) w_{3}^{(k+1)}: G\right)
$$

(and so completing the proof of Proposition (2.13)), will follow if I demonstrate that $\gamma_{k+1}^{q}=\delta_{k+1}$. This proof in effect repeats the above argument, with evaluations in $\Gamma$ instead of $G$ : using the associativities assured by construction,

Next,

$$
\begin{aligned}
\tilde{\mu}^{0}\left(\delta_{k+1}, \alpha_{k+1}\right)=\alpha_{k+2} & =\tilde{\mu}^{0}\left(\alpha_{k+1}, \gamma_{k+1}\right) \\
& \left.=\tilde{\mu}^{0}\left(\tilde{\mu}^{0}\left(\alpha_{j(q)}, \rho_{k-j(q)}^{q}\right)\right), \gamma_{k+1}\right) \\
& =\tilde{\mu}^{0}\left(\alpha_{j(q)}, \tilde{\mu}^{0}\left(\rho_{k-j(q)}^{q}, \gamma_{k+1}\right)\right) \\
& =\tilde{\mu}^{0}\left(\alpha_{j(q)}, \tilde{\mu}^{0}\left(\gamma_{k+1}^{0}, \rho_{k-j(q)}^{q}\right)\right) \\
& =\tilde{\mu}^{0}\left(\tilde{\mu}^{0}\left(\alpha_{j(q)}, \gamma_{k+1}^{0}\right), \rho_{k-j(q)}^{q}\right) .
\end{aligned}
$$

$$
\begin{aligned}
\tilde{\mu}^{0}\left(\alpha_{j(q)}, \gamma_{k+1}^{0}\right) & =\tilde{\mu}^{0}\left(\tilde{\mu}^{0}\left(\alpha_{j(q-1)}, \beta_{q-1}\right), \gamma_{k+1}^{0}\right) \\
& =\tilde{\mu}^{0}\left(\alpha_{j(q-1)}, \tilde{\mu}^{0}\left(\beta_{q-1}, \gamma_{k+1}^{0}\right)\right) \\
& =\tilde{\mu}^{0}\left(\tilde{\mu}^{0}\left(\alpha_{j(q-1)}, \gamma_{k+1}^{1}\right), \beta_{q-1}\right)
\end{aligned}
$$

and, by induction as before, passing $\gamma_{k+1}$ over the $\beta$ 's one by one,

$$
\begin{aligned}
\alpha_{k+2} & \left.\left.=\tilde{\mu}^{0}\left(\tilde{\mu}^{0}\left(\ldots \tilde{\mu}^{0}\left(\gamma_{k+1}^{q}, \beta_{0}\right), \beta_{1}\right), \ldots\right), \beta_{q-1}\right), \rho_{k-j(q)}^{q}\right) \\
& \left.\left.=\tilde{\mu}^{0}\left(\tilde{\mu}^{0}\left(\ldots \tilde{\mu}^{0}\left(\gamma_{k+1}^{q}, \tilde{\mu}^{0}\left(\beta_{0}, \beta_{1}\right)\right), \beta_{2}\right), \ldots\right), \beta_{q-1}\right), \rho_{k-j(q)}^{q}\right) \\
& \left.\left.=\tilde{\mu}^{0}\left(\tilde{\mu}^{0}\left(\ldots \tilde{\mu}^{0}\left(\gamma_{k+1}^{q}, \alpha_{j(2)}\right), \beta_{2}\right), \ldots\right), \beta_{q-1}\right), \rho_{k-j(q)}^{q}\right) \\
& =\tilde{\mu}^{0}\left(\tilde{\mu}^{0}\left(\ldots \tilde{\mu}^{0}\left(\gamma_{k+1}^{q}, \tilde{\mu}^{0}\left(\alpha_{j(2)}, \beta_{2}\right)\right), \beta_{3}\right), \ldots\right)
\end{aligned}
$$


and ultimately

$$
=\tilde{\mu}^{0}\left(\tilde{\mu}^{0}\left(\gamma_{k+1}^{q}, \alpha_{j(q)}\right), \rho_{k-j(q)}^{q}\right)=\tilde{\mu}^{0}\left(\gamma_{k+1}^{q}, \alpha_{k+1}\right) .
$$

(It should be observed that each associativity has indeed been arranged in the construction.) But therefore the definition of $\delta_{k+1}$ means that $\delta_{k+1}=\gamma_{k+1}^{q}$, as required.

Note that the assumption that $p(0)=e$ is not used in (2.14), but is essential in $(2.15)$ to start the induction.

(2.16) Proposition. Suppose $\Gamma, \tilde{\Gamma}, \Gamma^{0}, \tilde{\Gamma}^{0}, G, g, \tilde{U}$ are as in (2.13). Let $p_{0}$ and $p_{1}$ be homotopic paths from e to $x$ in $\tilde{V}^{0}$, where $x \in V^{0}$; let $w_{0}$ and $w_{1}$ be the right direct words associated with fine $\tilde{U}$-approximations of $p_{0}, p_{1}$ respectively. Then

$$
E\left(M(g) w_{0}: G\right)=E\left(M(g) w_{1}: G\right) .
$$

PROOF. By (2.13) it suffices to exhibit any fine U-approximations of $p_{0}$ and $p_{1}$ for which the associated direct words give the stated equality. Let $P: I \times I \rightarrow \widetilde{V}^{0}$ be a homotopy betueen $p_{0}$ and $p_{1}$, so that $P(t, 0)=p_{0}(t), P(t, 1)=p_{1}(t), P(0, t)=e$, $P(1, t)=x$ for all $t \in I$. Choose $\tilde{U}_{0}$ to be a $W\left(P(I \times I),\{e\}, \tilde{V}^{0}, \tilde{U}, \tilde{\Gamma}^{0}\right)$. Now choose $0=s_{0}<s_{1}<s_{2}<\ldots<s_{m}=1,0=t_{0}<t_{1}<\ldots<t_{m}=1$ so that, for each $(i, j)$ with $0 \leqslant i, j<m$, there is some $\left(\sigma_{i, j}, \tau_{i, j}\right) \in I \times I$ with

$$
P\left(\left[t_{i}, t_{i+1}\right] \times\left[s_{j}, s_{j+1}\right]\right) \subseteq \operatorname{int}_{\tilde{V}^{0}}\left\{\tilde{\mu}^{0}\left(P\left(\sigma_{i, j}, \tau_{i, j}\right) \times \tilde{U}_{0}\right)\right\},
$$

and select

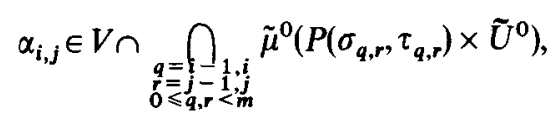

with the agreement that for all $j, \alpha_{0, j}=e$ and $\alpha_{m, j}=x$. Then $\left(\left(t_{i}, \alpha_{i, 0}\right)\right)_{0 \leqslant i \leqslant m}$ is a fine $\tilde{U}$ approximation of $p_{0}$ in $V^{0}$, and $\left(\left(t_{i}, \alpha_{i, m}\right)\right)_{0 \leqslant i \leqslant m}$ of $p_{1}$. Let the associated right direct words be $w_{0}, w_{1}$.

Define $\beta_{i, j}$ and $\gamma_{i, j}$ (for the relevant $i, j$ ) by taking them to be the unique elements of $\tilde{U} \cap V^{0} \subseteq V$ such that

$$
\alpha_{i+1, j}=\tilde{\mu}^{0}\left(\alpha_{i, j}, \beta_{i, j}\right), \quad \alpha_{i, j+1}=\tilde{\mu}^{0}\left(\alpha_{i, j}, \gamma_{i, j}\right) .
$$

Notice that $\gamma_{0, j}=\gamma_{m, j}=e$ for all $j$.

Now by associativity compare (2.13))

$$
\mu\left(\beta_{i, j}, \gamma_{i+1, j}\right)=\mu\left(\gamma_{i, j}, \beta_{i, j+1}\right) \text { for } 0 \leqslant i, j<m .
$$

Next, for $0 \leqslant j<m, 1 \leqslant i<m$, set

$$
\left.\left.\left.\left.v_{i, j}=\left(\left(\ldots\left(\beta_{0, j}, \beta_{1, j}\right), \ldots\right), \beta_{i-1, j}\right), \gamma_{i, j}\right), \beta_{i, j+1}\right), \ldots\right), \beta_{m-1, j+1}\right)
$$


and

$$
\begin{aligned}
& \left.v_{m, j}=\left(\left(\ldots\left(\beta_{0, j}, \beta_{1, j}\right), \beta_{2, j}\right), \ldots\right), \beta_{m-1, j}\right) \text { for } 0 \leqslant j \leqslant m, \\
& \left.v_{0, j}=\left(\left(\ldots\left(\gamma_{0, j}, \beta_{0, j+1}\right), \beta_{1, j+1}\right), \ldots\right), \beta_{m-1, j+1}\right) \text { for } 0 \leqslant j<m .
\end{aligned}
$$

Then, if $0 \leqslant j<m$ and $1 \leqslant i<m-1$,

$$
\begin{aligned}
\left.E\left(M(g) v_{i, j}: G\right)=E\left(M(g)\left(\left(\ldots\left(\beta_{0, j}, \beta_{1, j}\right), \ldots\right), \beta_{i-1, j}\right)\right): G\right) \cdot g\left(\gamma_{i, j}\right) & \\
& . g\left(\beta_{i, j+1}\right) . E\left(M(g)\left(\left(\ldots\left(\beta_{i+1, j+1}, \ldots\right), \beta_{m-1, j+1}\right): G\right)\right.
\end{aligned}
$$

and

$$
g\left(\gamma_{i, j}\right) \cdot g\left(\beta_{i, j+1}\right)=g\left(\mu\left(\gamma_{i, j}, \beta_{i, j+1}\right)\right)=g\left(\beta_{i, j}\right) \cdot g\left(\gamma_{i+1, j}\right),
$$

so that

$$
E\left(M(g) v_{i, j}: G\right)=E\left(M(g) v_{i+1, j}: G\right) \text { for } 0 \leqslant i<m-1 .
$$

When $i=m-1$ one has likewise (since $\gamma_{m, j}=e$ )

$$
E\left(M(g) v_{m-1, j}: G\right)=E\left(M(g)\left(v_{m, j}, \gamma_{m, j}\right): G\right)=E\left(M(g) v_{m, j}: G\right) .
$$

Finally,

$$
E\left(M(g) v_{o, j}: G\right)=E\left(M(g) v_{m, j+1}: G\right) \quad \text { for } 0 \leqslant j<m \text {, as } \gamma_{0, j}=e .
$$

Thus $E\left(M(g) v_{i, j}: G\right)$ is independent of $i$ and $j$; as $w_{0}=v_{m, 0}$ and $w_{1}=v_{m, 0}$, this proves the result.

(2.17) Proposition. Suppose $\Gamma, \tilde{\Gamma}, \Gamma^{0}, \tilde{\Gamma}^{0}, G, g, \tilde{U}$ are as in (2.13). Let $q: I \rightarrow \tilde{R}^{0}$ be a path with $q(0)=e$ and $q(1)=a \in R^{0}$. Let $v, w$ be respectively the left and right direct words associated with fine $\tilde{U}$-approximations of $q$ and $\tilde{v}^{0} \circ q$ in $V^{0}$. Then

$$
E(M(g) v: G)=E(M(g) w: G)^{-1} .
$$

ProOF. By (2.13) it is only necessary to prove this for a particular choice of fine $\tilde{U}$ approximations. Let $\tilde{W}$ be simultaneously a $W\left(q(I),\{e\}, \tilde{V}^{0}, \tilde{N}, \tilde{\Gamma}^{0}\right)$ and a $W\left(\tilde{v}^{0} \circ q(I),\{e\}, \tilde{V}^{0}, \tilde{N}, \tilde{\Gamma}^{0}\right), \quad$ where $\tilde{N} \subseteq \tilde{U} \quad$ is a $D\left(q(I), \Gamma, \tilde{\Gamma}^{0}\right)$ and a $W\left(q(I),\{e\}, \widetilde{V}^{0}, \tilde{U}, \tilde{\Gamma}^{0}\right)$. Now choose $0=t_{0}<t_{1}<\ldots<t_{m}=1$ so that for each $i$ there is $s_{i} \in I$ with

$$
q\left[t_{i}, t_{i+1}\right] \subseteq \operatorname{int}_{\widetilde{V}^{0}}\left\{\tilde{\mu}^{0}\left(q\left(s_{i}\right) \times \tilde{W}\right\} \cap \operatorname{int}_{\tilde{V}^{0}}\left\{\tilde{\mu}^{0}\left(\tilde{W} \times q\left(s_{i}\right)\right)\right\} .\right.
$$

Let $\alpha_{0}=e, \alpha_{m}=a$, and for $0<i<m$ select

$$
\alpha_{i} \in V^{0} \cap \tilde{\mu}^{0}\left(q\left(s_{i-1}\right) \times \tilde{W}\right) \cap \tilde{\mu}^{0}\left(q\left(s_{i}\right) \times \tilde{W}\right) \cap \tilde{\mu}^{0}\left(\tilde{W} \times q\left(s_{i-1}\right)\right) \cap \tilde{\mu}^{0}\left(\tilde{W} \times q\left(s_{i}\right)\right) .
$$

Thus $\left(\left(t_{i}, \alpha_{i}\right)\right)_{0 \leqslant i \leqslant m}$ is a fine $\tilde{U}$-approximation of $q$ in $V^{0}$. Let the associated left direct word be

$$
v=\left(\beta_{m-1},\left(\beta_{m-2},\left(\ldots,\left(\beta_{2},\left(\beta_{1}, \beta_{0}\right)\right) \ldots\right)\right)\right)
$$


Now by construction compare the conditions (2.2)(i), (iii) and (2.1)(ii)) each $\alpha_{i} \in \tilde{\mathbf{R}}^{0}$. From (2.6) (taking $K=q(I)$ ) it follows that $\tilde{v}^{0}\left(\alpha_{i}\right)$ must lie in $V^{0}$ for each $i$. Also, by (2.5), for all $t \in I$,

$$
\tilde{v}^{0} \tilde{\mu}^{0}(\tilde{N} \times q(t))=\tilde{\mu}^{0}\left(\tilde{v}^{0} \circ q(t) \times \tilde{N}\right)
$$

However,

$$
\alpha_{i} \in V^{0} \cap \bigcap_{t \in\left[t_{i}-1, t_{i+1}\right]} \tilde{\mu}^{0}(\tilde{N} \times q(t))
$$

by construction (compare (2.11), (2.12)). Thus

$$
\tilde{v}^{0}\left(\alpha_{i}\right) \in V^{0} \cap \bigcap_{t \in\left[t_{i}-1, t_{i+1}\right]} \tilde{\mu}^{0}\left(\tilde{v}^{0} \circ q(t) \times \tilde{N}\right),
$$

so that $\left(\left(t_{i}, \tilde{v}^{0}\left(\alpha_{i}\right)\right)\right)$ is a fine $\tilde{U}$-approximation of $\tilde{v}^{0} \circ q$ in $V^{0}$.

Next, $\beta_{i} \in V$ (compare (2.12), (2.13)) and $\beta_{i} \in \tilde{\mu}^{0}(\tilde{W} \times \tilde{W}) \subseteq \tilde{N}$; hence by (2.2)(iv), $\beta_{i} \in R$. Consequently by (2.5)

$$
\begin{aligned}
\tilde{v}^{0}\left(\alpha_{i+1}\right)=\tilde{v}^{0} \tilde{\mu}^{0}\left(\beta_{i}, \alpha_{i}\right) & =\tilde{\mu}^{0}\left(\tilde{v}^{0}\left(\alpha_{i}\right), \tilde{v}^{0}\left(\beta_{i}\right)\right) \\
& =\tilde{\mu}^{0}\left(\tilde{v}^{0}\left(\alpha_{i}\right), v\left(\beta_{i}\right)\right),
\end{aligned}
$$

so that the right direct word associated with $\left(\left(t_{i}, \tilde{v}^{0}\left(\alpha_{i}\right)\right)\right)$ is

$$
w=\left(\left(\left(\ldots\left(v\left(\beta_{0}\right), v\left(\beta_{1}\right)\right), v\left(\beta_{2}\right)\right), \ldots\right), v\left(\beta_{m-1}\right)\right) .
$$

But now

$$
\begin{aligned}
E(M(g) w: G) & =g\left(v\left(\beta_{0}\right)\right) \cdot g\left(v\left(\beta_{1}\right)\right) \ldots . g\left(v\left(\beta_{m-1}\right)\right) \\
& =\left(g\left(\beta_{0}\right)\right)^{-1} \cdot\left(g\left(\beta_{1}\right)\right)^{-1} \ldots .\left(g\left(\beta_{m-1}\right)\right)^{-1} \\
& =(E(M(g) v: G))^{-1}
\end{aligned}
$$

as required.

\section{The main argument}

(3.1) Proposition. Let an array of local groups as in (2.7) satisfy the further conditions

(i) that $\Gamma$ is full in $\tilde{\Gamma}$ and $\Gamma^{2}$ replete in $\tilde{\Gamma}^{2}$,

(ii) that $\tilde{V}$ is open in $\tilde{V}^{2}$ and $V=\tilde{V} \cap V^{2}$,

(iii) that $V$ is path-connected in $\tilde{V}$ (compare (2.9)),

(iv) that $\Gamma^{0}$ is convex in $\left.\tilde{\Gamma}^{0}, \tilde{\Gamma}^{1}, \tilde{\Gamma}^{2}\right)$ (compare (2.8)).

Suppose that $G$ is a group and $g: \Gamma \rightarrow G$ a homomorphism; let $\tilde{U}$ be a neighbourhood of $e$ in $\tilde{V}$ such that $\tilde{U} \times \tilde{U} \subseteq \tilde{Q}$. 
Then, given any $w \in M(\Gamma) \cap{ }^{e} M\left(\Gamma^{0}\right)$, there is a path $p_{w}: I \rightarrow \widetilde{V}^{0}$ such that $p_{w}(0)=e$, $p_{w}(1)=E\left(w: \Gamma^{0}\right)$, and, if $v$ is any fine $\tilde{U}$-approximation of $p_{w}$ in $V^{0}$, $E(M(g) v: G)=E(M(g) w: G)$.

Proof. Notice first that axioms (1.1)(IX) ensure $\tilde{V}^{0}, \tilde{V}^{1}$ are open in $\tilde{V}^{2}$, since $\tilde{V}$ is. The construction of $p_{w}$ proceeds by induction on the order of $w$ (cf. (1.6)). When the order is 0 , the constant path at $e$ satisfies the requirements.

Next, suppose $w=(a) \in M_{1}(\Gamma) \cap{ }^{e} M\left(\Gamma^{0}\right)$. By (iii), there is a path from $e$ to $a$ in $\tilde{V}$. Let $p_{w}$ be such a path. A fine $\tilde{U}$-approximation of $p_{w}$ in $V$ is automatically a fine $\tilde{U}$ approximation in $V^{0}$ (cf. (2.11)), and the associated right direct word $w^{\prime}$ is evaluable in $\tilde{\Gamma}$ to $a$, by definition. As $\Gamma$ is full in $\tilde{\Gamma}$ by $(i), w^{\prime}$ is also evaluable in $\Gamma$ to $a$. Since $g$ is a homomorphism,

$$
E\left(M(g) w^{\prime}: G\right)=g\left(E\left(w^{\prime}: \Gamma\right)\right)=g(a)
$$

as required.

Now suppose $w=\left(w_{1}, w_{2}\right)$, where $w_{1}, w_{2}$ are of strictly lower order than $w$, so that $p_{w_{1}}, p_{w_{2}}$ are already defined. Recall that $w \in{ }^{e} M\left(\Gamma^{0}\right)$; thus $\left(p_{w_{1}}(1), p_{w_{2}}(1)\right) \in Q^{0}$. By (iv) (see (2.8)) there is a path $p_{w_{2}}^{\prime}: I \rightarrow \tilde{V}^{0}$ such that

$$
p_{w_{1}}(1) \times p_{w_{2}}^{\prime}(I) \subseteq \tilde{Q}^{1} \text {. }
$$

This means in turn that $p_{w_{1}}$ and $p_{w_{2}}^{\prime}$ satisfy the hypotheses of (2.8)(ii), and consequently there is a path $p_{w}: I \rightarrow \tilde{V}^{0}$ homotopic in $\tilde{V}^{2}$ (with fixed end-points) to the path $r_{w}$, where

$$
\begin{aligned}
& r_{w}(t)=p_{w_{1}}(2 t) \quad \text { when } 0 \leqslant t \leqslant \frac{1}{2}, \\
& r_{w}(t)=\tilde{\mu}^{1}\left(p_{w_{1}}(1), p_{w_{2}}^{\prime}(2 t-1)\right) \text { when } \frac{1}{2} \leqslant t \leqslant 1 .
\end{aligned}
$$

Let $\tilde{W}$ be simultaneously a $\left.W r_{w}(I),\{e\}, \tilde{V}^{2}, \tilde{U}, \tilde{\Gamma}^{2}\right)$ and a

$$
W\left(\left\{p_{w_{1}}(1)\right\}, p_{w_{2}}(I), \tilde{V}^{2}, \tilde{U}, \tilde{\Gamma}^{2}\right) \text {. }
$$

Let $\left(\left(t_{i}^{1}, \alpha_{i}^{1}\right)\right)_{0 \leqslant i \leqslant m}$ be a fine $\tilde{W}$-approximation of $p_{w_{1}}$ in $V^{0}$, with associated right direct word

$$
v_{1}=\left(\left(\left(\ldots\left(\beta_{0}^{1}, \beta_{1}^{1}\right), \beta_{2}^{1}\right), \ldots\right), \beta_{m-1}^{1}\right),
$$

and let $\left(\left(t_{i}^{2}, \alpha_{i}^{2}\right)\right)_{0 \leqslant i \leqslant n}$ be a fine $\tilde{W}$-approximation of $p_{w_{2}}^{\prime}$ in $V^{2}$, with associated right direct word

$$
v_{2}^{\prime}=\left(\left(\left(\ldots\left(\beta_{0}^{2}, \beta_{1}^{2}\right), \beta_{2}^{2}\right), \ldots\right), \beta_{n-1}^{2}\right) .
$$

Define $t_{i}=\frac{1}{2} t_{i}^{1}$ for $0 \leqslant i \leqslant m ; \quad t_{i}=\frac{1}{2}+\frac{1}{2} t_{i-m}^{2}$ for $m \leqslant i \leqslant m+n: \alpha_{i}=\alpha_{i}^{1}$ for $0 \leqslant i \leqslant m ; \quad \alpha_{i}=\tilde{\mu}^{2}\left(\alpha_{m}^{1}, \alpha_{i-m}^{2}\right)$ for $m \leqslant i \leqslant m+n$. (Note that $\left(\alpha_{m}^{1}, \alpha_{i-m}^{2}\right) \in \check{Q}^{2}$ by construction, since $\alpha_{m}^{1}=p_{w_{1}}(1)$ and $p_{w_{1}}(1) \times p_{w_{2}}(I) \subseteq \widetilde{Q}^{2}$. See (2.1). As $\alpha_{m}^{1}, \alpha_{i-m}^{2}$ are in $V^{2}$, and $\Gamma^{2}$ is replete in $\tilde{\Gamma}^{2}$, it follows that $\alpha_{i} \in V^{2}$.) By this construction $I$ have 
ensured, via (2.1)(ii), (v), that the new sequence $\left(\left(t_{i}, \alpha_{i}\right)\right)_{0 \leqslant i \leqslant m+n}$ is a fine $\tilde{U}$ approximation of $r_{w}$ in $V^{2}$, with associated right direct word

$$
v^{\prime}=\left(\left(\left(\ldots\left(\beta_{0}, \beta_{1}\right), \beta_{2}\right), \ldots\right), \beta_{m+n-1}\right),
$$

where $\beta_{i}=\beta_{i}^{1}$ for $0 \leqslant i \leqslant m-1$ and $\beta_{i}=\beta_{i-m}^{2}$ for $m \leqslant i \leqslant m+n-1$. However, it is clear from this that

$$
E\left(M(g) v^{\prime}: G\right)=E\left(M(g) v_{1}: G\right) . E\left(M(g) v_{2}^{\prime}: G\right) .
$$

Let $v$ be the right direct word associated with a fine $\tilde{U}$-approximation of $p_{w}$ in $V^{0}$, which will also be a fine $\tilde{U}$-approximation of $p_{w}$ in $V^{2}$ (cf. (2.11)). Thus, by (2.16),

$$
E(M(g) v: G)=E\left(M(g) v^{\prime}: G\right) .
$$

Let $v_{2}$ be the right direct word associated with a fine $\tilde{U}$-approximation of $p_{w_{2}}$ in $V^{0}$; it will also be a fine $\tilde{U}$-approximation in $V^{2}$. By (2.16) again,

$$
E\left(M(g) v_{2}: G\right)=E\left(M(g) v_{2}^{\prime}: G\right) .
$$

Hence

$$
\begin{aligned}
E(M(g) v: G) & =E\left(M(g) v_{1}: G\right) \cdot E\left(M(g) v_{2}^{\prime}: G\right) \\
& =E\left(M(g) v_{1}: G\right) \cdot E\left(M(g) v_{2}: G\right) \\
& =E\left(M(g) w_{1}: G\right) \cdot E\left(M(g) w_{2}: G\right) \quad(\text { by the inductive hypothesis) } \\
& =E\left(M(g)\left(w_{1}, w_{2}\right): G\right) \\
& =E(M(g) w: G),
\end{aligned}
$$

as required : so $p_{w}$ has been defined satisfactorily.

Finally, suppose $w=\left(w_{0}\right)^{-1}$, where $p_{w_{0}}$ has been defined (more formally, suppose $w=\left(w_{1}, w_{2}\right)^{-1}$, where $p_{\left(w_{1}, w_{2}\right) \pm}$, has already been defined). Take $p=p_{w_{0}}$ in (2.8)(iii); certainly $p_{w_{0}}(1) \in R^{0}$ as there required, since $w \in{ }^{e} M\left(\Gamma^{0}\right)$. Thus I obtain paths $q: I \rightarrow \widetilde{R}^{2}$ and $r: I \rightarrow \tilde{V}^{0}$. Set $p_{w}=r$. Then, by definition,

$$
p_{w}(0)=e, p_{w}(1)=v^{0}\left(E\left(w_{0}: \Gamma\right)\right)=E(w: \Gamma) .
$$

Suppose $v_{0}$ is the right direct word associated with a fine $\tilde{U}$-approximation of $p_{w_{0}}$ in $V^{0}$, and let $v$ be similarly associated with a fine $\tilde{U}$-approximation of $p_{w}$ in $V^{0}$. (These are also approximations in $V^{2}$.) Let $v_{1}, v_{2}$ be respectively right and left direct words associated with fine $\tilde{U}$-approximations of $q$ and of $\tilde{v}^{2} \circ q$ in $V^{2}$. Then

$$
\begin{aligned}
E\left(M(g) v_{0}: G\right) & =E\left(M(g) v_{1}: G\right) & & \text { by }(2.16), \\
E\left(M(g) v_{1}: G\right) & =E\left(M(g) v_{2}: G\right)^{-1} & & \text { by }(2.17), \\
E(M(g) v: G) & =E\left(M(g) v_{2}: G\right) & & \text { by }(2.16) .
\end{aligned}
$$


Hence

$$
\begin{aligned}
E(M(g) v: G) & =E\left(M(g) v_{0}: G\right)^{-1} \\
& =E\left(M(g) w_{0}: G\right)^{-1} \text { by hypothesis, } \\
& =E(M(g) w: G)
\end{aligned}
$$

This completes the inductive construction of $p_{w}$ (cf. (1.6)).

(3.2) THEOREM. Suppose the hypotheses of (3.1) are satisfied, and also

(v) $\tilde{V}^{0}$ is simply-connected in $\tilde{V}^{2}$ (as in (2.9)).

Let $\Delta$ be the portion of $\Gamma^{0}$ whose underlying subspace of $V^{0}$ is

$$
Y=E\left(M(\Gamma) \cap{ }^{e} M\left(\Gamma^{0}\right): \Gamma^{0}\right) \text {. }
$$

Then there is a unique extension of $g$ to a homomorphism $\tilde{g}: \Delta \rightarrow G$. In particular, if $\tilde{V}^{0}$ is connected and $V^{0}=V^{2} \cap \tilde{V}^{0}$, then $g$ extends uniquely to a homomorphism $\tilde{g}: \Gamma^{0} \rightarrow G$.

Note. $V$ is open in $V^{0}$ by (3.1)(ii). Hence, applying (1.1)(IX) to $\Gamma^{0}$, $E\left(M(\Gamma) \cap{ }^{e} M\left(\Gamma^{0}\right): \Gamma^{0}\right)$ is open in $V^{0}$, and, by (1.3), $\Delta$ is well-defined.

Proof. Let $w_{1}, w_{2} \in M(\Gamma) \cap{ }^{e} M\left(\Gamma^{0}\right)$, and suppose

$$
E\left(w_{1}: \Gamma^{0}\right)=x=E\left(w_{2}: \Gamma^{0}\right) .
$$

Take paths $p_{w_{1}}, p_{w_{2}}$ as in (3.1). They join $e$ to $x$ in $\tilde{V}^{0}$, and so are homotopic, with fixed end-points, in $\tilde{V}^{2}$. Let $v_{1}, v_{2}$ be right direct words associated with fine $\tilde{U}$ approximations in $V^{0}$ of $p_{w_{1}}, p_{w_{2}}$ respectively; they are automatically fine $\tilde{U}$ approximations of these paths in $V^{2}$ as well. By (3.1),

$$
E\left(M(g) w_{1}: G\right)=E\left(M(g) v_{1}: G\right), \quad E\left(M(g) w_{2}: G\right)=E\left(M(g) v_{2}: G\right) .
$$

But, by (2.16),

$$
E\left(M(g) v_{1}: G\right)=E\left(M(g) v_{2}: G\right) .
$$

Consequently I may define unambiguously: $\tilde{g}(x)=E(M(g) w: G)$ for any $w \in M(\Gamma) \cap{ }^{e} M\left(\Gamma^{0}\right)$ such that $E\left(w: \Gamma^{0}\right)=x$. If $\tilde{g}$ is to be a homomorphism, this is the only possible value for $\tilde{g}(x)$. It only remains to show that $\tilde{g}$, so defined, is indeed a homomorphism.

First, if $x=E\left(w: \Gamma^{0}\right) \in R^{0}$, where $w \in M(\Gamma) \cap{ }^{e} M\left(\Gamma^{0}\right)$, then

$$
v^{0}(x)=E\left((w, \phi)^{-1}: \Gamma^{0}\right),
$$


and consequently

$$
\tilde{g}\left(v^{0}(x)\right)=E\left(M(g)(w, \phi)^{-1}: G\right)=E(M(g) w: G)^{-1}=(\tilde{g}(x))^{-1} .
$$

Similarly, if $\left(x_{1}, x_{2}\right) \in Q^{0}$, and $x_{i}=E\left(w_{i}: \Gamma^{0}\right)$ for $i=1,2$, then

$$
\mu^{0}\left(x_{1}, x_{2}\right)=E\left(\left(w_{1}, w_{2}\right): \Gamma^{0}\right),
$$

and so

$$
\begin{aligned}
\tilde{g}\left(\mu^{0}\left(x_{1}, x_{2}\right)\right) & =E\left(M(g)\left(w_{1}, w_{2}\right): G\right)=E\left(M(g) w_{1}: G\right) . E\left(M(g) w_{2}: G\right) \\
& =\tilde{g}\left(x_{1}\right) \cdot \tilde{g}\left(x_{2}\right),
\end{aligned}
$$

as required. The final assertion of the theorem follows by noting that, if it is known that $V^{0}=V^{2} \cap \tilde{V}^{0}$ and that $\Gamma^{2}$ is replete in $\tilde{\Gamma}^{2}$, then $\Gamma^{0}$ is replete in $\tilde{\Gamma}^{0}$; hence connectedness of $\tilde{V}^{0}$, and the fact that $\tilde{V}$ is open in $\tilde{V}^{0}$, show by (2.4) that $\Delta=\Gamma^{0}$.

\section{Conclusions and comments}

(4.1) Definition. Let $\Gamma$ be a local group. The universal group of $\Gamma$, written $U(\Gamma)$, is the group generated by the elements of $V$, with relations

$$
x . v(x)=e \quad \text { for } x \in R, \quad x \cdot y=\mu(x, y) \text { for }(x, y) \in Q .
$$

That is, $U(\Gamma)=G(\Gamma) / N$, where $N$ is the smallest normal subgroup of $G(\Gamma)$ containing all the terms $(x, v(x))$ for $x \in R$ and $\left(y^{-1}, x^{-1}, \mu(x, y)\right)$ for $(x, y) \in Q$.

There is an obvious canonical map $U_{\Gamma}: \Gamma \rightarrow U(\Gamma): x \mapsto(x) N$, which the definition of $N$ makes a homomorphism, and which is characterized up to natural isomorphism by the following property : given any homomorphism $g: \Gamma \rightarrow G$, for any group $G$, there is a unique homomorphism of groups $\bar{g}: U(\Gamma) \rightarrow G$ such that $\bar{g} \circ U_{\Gamma}=g$. It follows that, for each homomorphism $f: \Gamma \rightarrow \Delta$ of local groups, there is a naturally induced homomorphism of groups

$$
U(f): U(\Gamma) \rightarrow U(\Delta),
$$

and that $U$ is thus a functor from local groups to groups. The homomorphism $U(f)$ will be an isomorphism if and only if, given any group $G$ and any homomorphism $g: \Gamma \rightarrow G$, there is a unique homomorphism $\hat{g}: \Delta \rightarrow G$ such that $\hat{g} \circ f=g$.

(4.2) THEOREM. Suppose $\Gamma$ is a dense replete local subgroup of $\tilde{\Gamma}$, and $\tilde{A} \subseteq \tilde{A}_{0} \subseteq \tilde{A}_{1}$ are path-connected open neighbourhoods of e in $\tilde{V}$ such that $\tilde{A}_{0} \subseteq \tilde{R}$, $\tilde{v}\left(\tilde{A}_{0}\right) \subseteq \tilde{A}_{1}, \tilde{A}_{0} \times \tilde{A}_{0} \subseteq \tilde{Q}$, and $\tilde{\mu}\left(\tilde{A}_{0} \times \tilde{A}_{0}\right) \subseteq \tilde{A}_{1} ;$ and let $\tilde{A}_{1}$ be simply-connected in $\tilde{V}$. Then, for any group $G$ and any homomorphism $g: \Gamma \mid \tilde{A} \cap V \rightarrow G$, there is a unique 
extension of $g$ to a homomorphism $g_{0}: \Gamma \mid \tilde{A}_{0} \cap V \rightarrow G$. Equivalently, the inclusion homomorphism of local groups, $i: \Gamma|\tilde{A} \cap V \rightarrow \Gamma| \tilde{A}_{0} \cap V$, induces an isomorphism of universal groups, $U(i): U(\Gamma \mid \tilde{A} \cap V) \rightarrow U\left(\Gamma \mid \tilde{A}_{0} \cap V\right)$.

Proof. Apply (3.2) to the array

$$
\begin{aligned}
& \tilde{\Gamma}|\tilde{A} \subseteq \tilde{\Gamma}| \tilde{A}_{0} \subseteq \tilde{\Gamma} \mid \tilde{A}_{1} \subseteq \tilde{\Gamma} \\
& \text { UI UI UI UI } \\
& \Gamma|\tilde{A} \cap V \subseteq \Gamma| \tilde{A}_{0} \cap V \subseteq \Gamma \mid \tilde{A}_{1} \cap V \subseteq \Gamma
\end{aligned}
$$

The hypotheses of (3.2) are satisfied (see (2.10)(iv), and note that repleteness of $\Gamma$ in $\tilde{\Gamma}$ trivially entails fullness of $\Gamma \mid \tilde{A} \cap V$ in $\tilde{\Gamma} \mid \tilde{A}$ ). Thus the result follows from (3.2) and (4.1).

(4.3) CoRollary. Suppose $\tilde{H}$ is a connected and simply-connected topological group and $H$ a dense subgroup. Let $\tilde{V}$ be a path-connected open neighbourhood of $e$ in $\tilde{H}$. Then, given any group $G$ and homomorphism $g: H \mid \tilde{V} \cap H \rightarrow G$, there is a unique homomorphism $\tilde{g}: H \rightarrow G$ extending $g$, and, if $i: H \mid \tilde{V} \cap H \rightarrow H$ is the inclusion homomorphism, the induced homomorphism

$$
\vec{\imath}: U(H \mid \tilde{V} \cap H) \rightarrow H
$$

is an isomorphism of groups.

Proof. Set $\tilde{A}_{0}=\tilde{A}_{1}=\tilde{H}$ in (4.2), and note that $U(H)$ is naturally identified with H.

(4.4) Corollary. Let $\Gamma$ be a simply-connected and locally path-connected local group. Suppose $A_{1}, A_{2}$ are open connected neighbourhoods of $e$ in $V$ such that, for $i=1,2$,

$$
A_{i} \subseteq R, \quad A_{i} \times A_{i} \subseteq Q
$$

Then $U\left(\Gamma \mid A_{1}\right)$ and $U\left(\Gamma \mid A_{2}\right)$ are canonically isomorphic.

ProOF. Choose a connected open neighbourhood $A$ of $e$ included in both $A_{1}$ and $A_{2}$. Then by (4.2) (taking $\Gamma=\tilde{\Gamma}$ and $\tilde{A}_{0}=\tilde{A}_{1}=V$ ) the homomorphisms $U(\Gamma \mid A) \rightarrow U\left(\Gamma \mid A_{i}\right)$ induced by inclusion are both isomorphisms.

(4.5) The local groups in (4.2), (4.3), and (4.4) need not be symmetric; this is a gain from the non-standard definition (1.1). Inversive fullness (see (1.3)) was not needed because of (2.6).

Note that these corollaries are indeed very much weaker than the basic but indigestible theorem (3.2). They are stated for portions : however, the 'natural' local 
group structure on, for instance, the subset $\left\{(x, y, z): 0<(x-1)^{2}+y^{2}+z^{2}<2\right\}$ in $\mathbf{R}^{3}$, is not that of a portion of $\mathbf{R}^{3}$, in which one could multiply across the deleted point. The general convexity condition (3.1)(iv) also has its uses - see (4.6) below.

In the obvious cases, such as rational subgroups of unitary groups, (4.3) is a weaker version (differing in certain formal respects) of a theorem of Ganea (1951). A second result of Ganea (1953) should be compared.

(4.6) Let $g$ be a real Banach Lie algebra : that is, a real Lie algebra furnished with a norm which is submultiplicative with respect to the Lie bracket and makes it a Banach space. Let $B(r)$ denote the open ball of radius $r$ about the origin ing. There are universal constants $k$ and $l$ such that the Campbell-Hausdorff formula makes $B(k)$ a local group (including $B(l)$ ) in which the product of any two elements of $B(l)$ is defined. (4.4) now states that all connected portions of $B(k)$ included in $B(l)$ have canonically isomorphic universal groups.

If $g$ derives from a connected Banach Lie group $(\mathfrak{b}$ (which need not be the case : see Serre (1965), p. LG 5.41), then the universal group of any sufficiently small connected portion of $B(k)$ is naturally identified with the universal cover of $(5$. In the general case, I believe that the essence of my proof cannot be simplified, although technical modifications are possible. For instance, $(2.11)-(2.17)$ may be reformulated in terms of the development in $\tilde{V}$ of paths in $g$ (a 'differential' rather than 'difference' argument); but such reasoning, using differential equations, requires completeness, and so is less general than mine. Suppose, for example, that $\mathfrak{b}$ is a non-closed subspace of $g$ including the algebraic commutator subalgebra $[g, g]$. Dynkin's form of the Campbell-Hausdorff formula defines a local group corresponding to $\mathfrak{h}$, despite the lack of completeness.

If $g$ comes from a simply-connected group (5, Lazard and Tits (1966) showed that $B(\pi)$ has a local group structure which embeds by the exponential in $\mathfrak{6}$. Swierczkowski (1971) pointed out that any Banach Lie algebra is the quotient (with quotient norm) of one that enlarges to a Lie group. It may be deduced from these facts that, in any Banach Lie algebra, $B(\pi)$ may be given the structure of a local group. Furthermore, if one employs the convexity hypotheses in (3.2), it follows that any connected portion of $B(\pi)$ has canonically isomorphic universal group. These assertions are not trivial.

(4.7) The theorem of Lazard and Tits also says that the question whether $g$ corresponds to a Lie group is already determined in $B(\pi)$. One may ask if there is a general result of this kind for local groups, and it is easy to see that this amounts to finding results such as (3.2) when $G$ is only a local group. Now I have freely exploited the hypothesis that $G$ is a group in all my arguments : $I$ have assumed that all products in $G$ are defined, and that they are all associative. It is fairly clear, however, 
that the restricted associativity of products given by (1.1)(VI) would suffice if we restricted attention to continuous homomorphisms of local groups. On the other hand, it seems difficult to ensure that all the products that are used in the proofs actually exist in $G$. One might introduce a suitable notion of path-length, and postulate that all homotopies used pass only through paths of appropriately bounded length; but both statement and proof of such a theorem seem better left to the imagination.

\section{References}

C. Chevalley, (1946). Theory of Lie groups, I (Princeton University Press, Princeton, N.J.).

T. Ganea (1951), 'Du prolongement des représentations locales des groupes topologiques', Acta Sci. Math. (Szeged) 14, 115-124.

T. Ganea (1953), 'Groupes topologiques sans centre', Rev. Univ. 'C.I.Parhon' Politehn. Bucuresti, Ser. Sti. Nat., no. 3, 37-38.

A. G. Kurosh (1960), Theory of groups, $I I$ (Chelsea, New York).

M. Lazard and J. Tits (1966), 'Domaines d'injectivité de l'application exponentielle', Topology 4, 315-322.

L. S. Pontrjagin (1939), Topological groups (Princeton University Press, Princeton, N.J.).

J.-P. Serre (1965), Lie algebras and Lie groups (Benjamin, New York).

S. Swierczkowski (1965), 'Embedding theorems for local analytic groups', Acta Math. 114, 207-235.

S. Swierczkowski (1971), 'The path functor on Banach Lie algebras', Indagationes Math. 33, 235-239.

Department of Mathematics

Victoria University of Wellington

Private Bag, Wellington

New Zealand 